\title{
Paraneoplastic systemic sclerosis associated with colorectal carcinoma
}

\author{
Dominik Samotij ${ }^{1}$, Joanna Maj' ${ }^{2}$ Adam Reich ${ }^{1}$ \\ ${ }^{1}$ Department of Dermatology, University of Rzeszow, Poland \\ ${ }^{2}$ Department of Dermatology, Venereology, and Allergology, Wroclaw Medical University, Poland
}

\begin{abstract}
A number of rheumatic disorders may appear as paraneoplastic syndromes, the most common being dermatomyositis or polymyositis. Systemic sclerosis is associated with a slightly increased risk of cancer, although its direct association with malignancies is controversial. We describe a case of a 57-year-old male with rectal adenocarcinoma and systemic sclerosis. Close temporal relationship between the initial presentation and parallel course of both conditions, as well as atypically rapid progression of systemic sclerosis symptoms, were observed in the reported case. The strict relation between these two conditions suggested that systemic sclerosis was a paraneoplastic syndrome rather than a concomitant morbidity in the presented patient. Current literature on systemic sclerosis coexisting with colorectal tumours is very limited, especially in the paraneoplastic setting.
\end{abstract}

Key words: systemic sclerosis, paraneoplastic syndrome, rectal carcinoma, rheumatic disorders, malignancy.

\section{Introduction}

Systemic sclerosis (SSc) is an uncommon connective tissue disease featured by microangiopathy and progressive fibrosis of the skin and internal organs. It is a clinically heterogeneous disorder associated with high mortality and morbidity related to internal organ failure and a lack of effective antifibrotic therapies. The pathogenesis of SSc is poorly understood. It is postulated that certain genetic factors influence the risk of the disease development and its clinical phenotype. It is generally accepted that vascular injury (i.e. small vessel vasculopathy and endothelial dysfunction), abnormal fibroblast activation resulting in abundant extracellular matrix production and excessive tissue fibrosis, along with immune system dysregulation, are distinguishing hallmarks of the disease [1].

An elevated age- and gender-adjusted risk of malignant disorder in SSc patients compared with their general population counterparts has been suggested by several epidemiological studies. Estimates of malignancies prevalence in all patients diagnosed with SSc range from 3 to $11 \%[2,3]$. A recent meta-analysis of a population-based cohort study performed by Onishi et al. [4] reported the pooled standardised incidence ratio for overall cancers in SSc at 1.41 (95\% confidence interval: 1.18-1.68), and significantly higher in males. Lung cancer was the most frequent type of malignancy diagnosed in SSc individuals followed by gallbladder and liver cancer, as well as various haematological malignancies [4].

In rare cases, SSc may present as a paraneoplastic syndrome (PS) defined as grouping of distinctive symptoms occurring in patients with malignant neoplasms related to the presence of cancer that cannot be readily explained by tumour-related mass effect consequent to a local invasion and/or distant metastases; also not dependent on metabolic and nutritional deficits, infections, coagulopathy, or adverse effects of anticancer treatment [5].

Here, we present a case of rapidly progressive SSC preceded by adenocarcinoma of the rectum. The direct time-relation between the diagnosis of malignancy and the initial symptoms of SSc, in addition to SSc progression directly preceding the cancer recurrence, were

Address for correspondence:

Dominik Samotij, Department of Dermatology, University of Rzeszow, 2 Fryderyka Szopena St., 35-055 Rzeszow, Poland,

e-mail: dominik.samotij@gmail.com

Submitted: 20.04.2018; Accepted: 24.05.2018 
highly suggestive of a paraneoplastic background of this rheumatic condition in the presented patient.

\section{Case report}

We describe a 57-year-old man with established diagnosis of rectal adenocarcinoma, who underwent a low anterior resection procedure with protective transverse colostomy with pre-operative radiochemotherapy and adjuvant chemotherapy. About eight weeks after the surgical treatment the patient was referred to the dermatology clinic with symptoms of sudden-onset SSc with rapid progression of clinical manifestations. Physical examination revealed marked thickening and hardening of the skin of the upper limbs, including sclerodactyly causing flexural contractures of the interphalangeal joints. In addition, hand stiffness, puffy fingers, and digital pitting scars were noted (Fig. 1). Skin hardening was also present, to a lesser extent, on the lower extremities, causing dermatogenic knee flexion contractures resulting in considerable immobilisation.

Shiny and smooth facial appearance was also clearly visible with typical skin hardening, limited mouth opening, microcheilia (Fig. 2A), and discrete mat-like telangiectasias on the forehead and cheeks (Fig. 2B).

Raynaud's phenomenon was induced through cold-water provocation test. Early scleroderma pattern was recognised in nailfold capillaroscopy. On the basis of the above findings, the patient fulfilled diagnostic criteria based on 2013 ACR/EULAR Classification Criteria for Systemic Sclerosis scoring, thus confirming the diagnosis of definitive SSC [6]. Further laboratory assessments and imaging were performed to detect visceral involvement and establish prognosis. High titres of antinuclear antibodies (1:1280) with nucleolar staining pattern were detected using indirect immunofluorescence technique on HEp-2 cells. However, using western-blot analysis we were unable to identify the specificity of these antibodies (The following antigens were tested: DSF 70, nRNP/Sm, Sm, SS-A, Ro-52, SS-B, Scl-70, PM-Scl, Jo-1, CENP B, PCNA, dsDNA, nucleosomes, histones, ribosomal $P$ protein, $A M A-M 2)$.

Chest high-resolution computed tomography demonstrated bibasilar pulmonary fibrosis with preserved pulmonary function as assessed by spirometry. Echocardiography was not suggestive of pulmonary arterial hypertension. Due to dyspeptic symptoms reported by the patient, such as heartburn, significant regurgitation, and solid-food dysphagia, oesophageal manometry was performed displaying reduced distal oesophageal contraction amplitude and hypotensive lower oesophageal sphincter - the typical pattern for SSc-related involvement.

Since the moment of establishing SSc diagnosis, regularly scheduled follow-up visits with treatment effica- cy evaluation have been performed in our department. Slow but gradual improvement in skin thickening was noted with neither further progression of interstitial lung disease nor impairment of physical exercise tolerance. Two years after the initial symptoms appeared, the patient was admitted to our department on an urgent basis with symptoms of rapid progression of SSc symptoms with concomitant episodes of fever, generalised malaise, and abdominal pain. Physical examination revealed nearly immobilising skin hardening of hands and lower extremities. Notably, the severity of cutaneous involvement was even more pronounced than we had observed at the moment of SSc diagnosis two years before. Due to the above-mentioned systemic symptoms the patient was referred to the Department of Oncology to rule out cancer reappearance; inoperable, locally advanced recurrence of primary tumour was diagnosed, and the patient was qualified for palliative care.

\section{Discussion}

Clear time relationship between cancer diagnosis and the initial presentation of SSc, combined with marked progression of SSc symptoms that had directly preced-

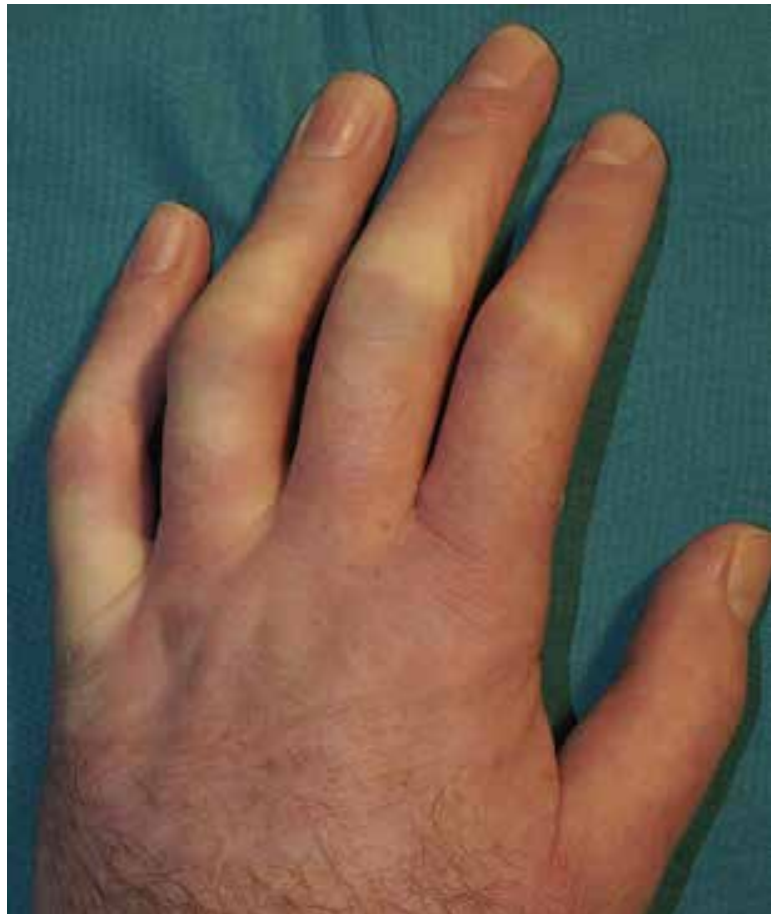

Fig. 1. Hardening and thickening of the skin proximal to metacarpophalangeal joints with sclerodactyly of the left hand resulting in flexural contractures of the proximal interphalangeal joints. Puffy fingers and macroscopically visible perfusion deficit is also seen. 

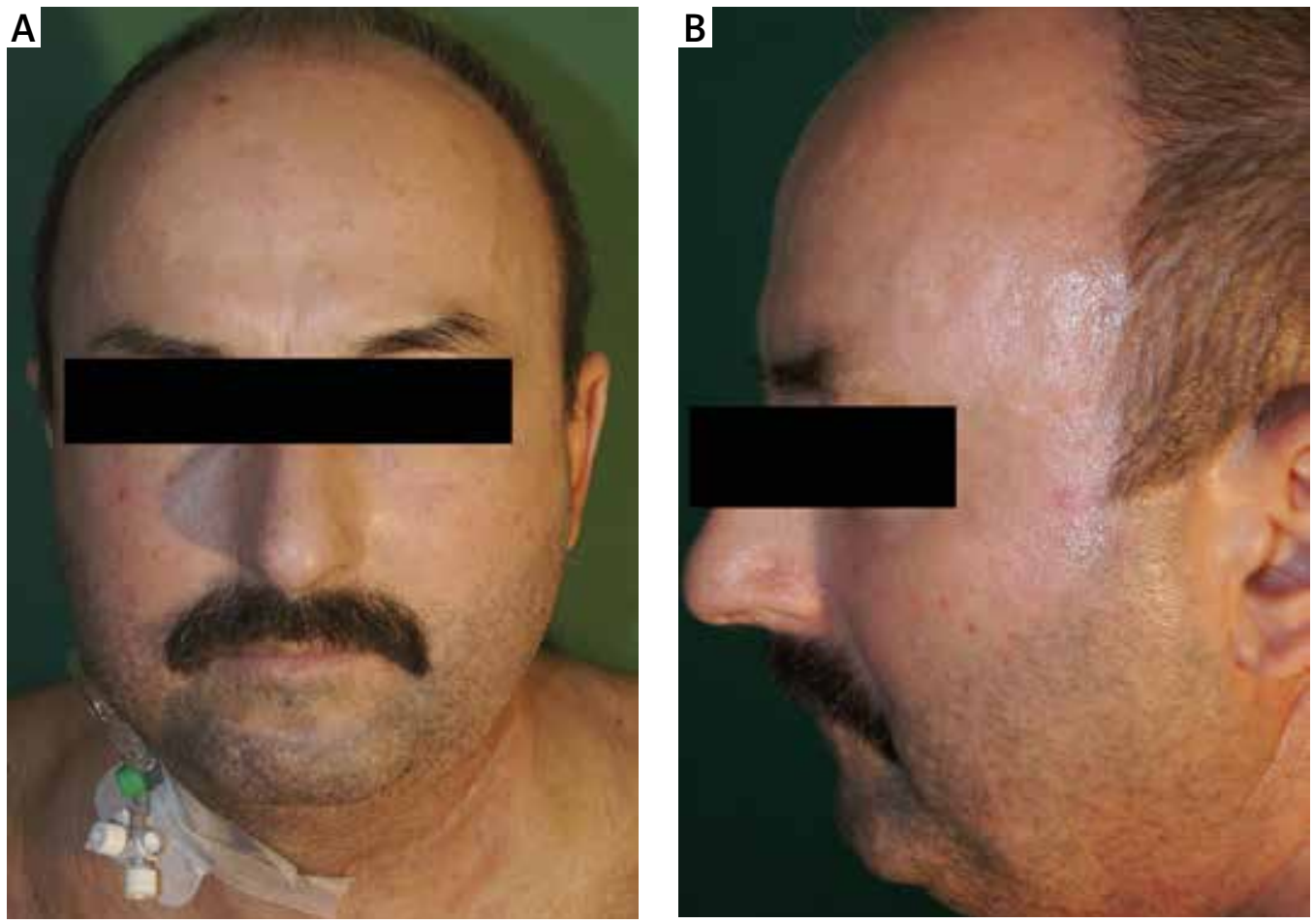

Fig. 2. (A) Shiny and taut appearance of the facial skin. A central venous catheter was placed as the peripheral venous access was impossible due to a significant skin hardening of the limbs. (B) Telangiectasias on the forehead and the left chin*.

*All pictures of the patient were taken after achieving written consent of the patient. The patient agreed to publish his pictures for scientific purposes.

ed tumour recurrence, suggested that it was not only a random temporal coexistence of these two entities, but SSc was in fact a paraneoplastic entity in this setting.

It is estimated that PSs affect up to $8 \%$ of patients with cancer; however, the actual prevalence is difficult to determine due to their rarity [5]. Collection of symptoms occurring in the course of PS may result from biologically functional substances produced by the tumour cells or cancer-induced autoimmunity. PSs are a highly heterogeneous group of disorders that affect diverse organs or organ systems, usually located remote and non-contiguous to the primary tumour and its metastases. PS-associated symptoms may be detected at a less advanced stage of cancer development, thus giving important clues to the underlying aetiology and making earlier diagnosis possible. Unfortunately, PSs are more frequently detected simultaneously or after a cancer diagnosis $[7,8]$. PSs are important from the clinical point of view because they present challenges to management requiring several therapeutic approaches of the cancer patient, and potentially lead to significant clinical problems that affect quality of life or may contribute to potentially lethal complications. In many cases, PS may mask some of the symptoms directly related to malignancy and hence contribute to a late cancer diagnosis. The majority of PSs are endocrine in nature, although haematological, neurological, and rheumatic symptoms are also reported [9]. The most common example of rheumatic PS is dermatomyositis/polymyositis [10].

A number of other rheumatic diseases may be triggered by underlying tumour such as SSc, as in the case described. It should be underlined that only a small proportion of all cases of rheumatic syndromes coexisting with carcinoma are of paraneoplastic nature. Sine qua non conditions for defining certain conditions as paraneoplastic is the time relation between the moment of cancer diagnosis and the onset of a certain disease. In addition, the clinical correlation between the progression of cancer and the severity of symptoms of a certain PS, including improvement or even resolution of its symptoms after efficacious antineoplastic treatment, is observed. Long-term follow-up of a patient and detailed analysis of a certain case is therefore necessary to establish if there is an association or only a random coexistence of two conditions. 
Certain differences in the clinical presentation and immunological profile between non-paraneoplastic autoimmune diseases and their paraneoplastic counterparts have been reported, but they are very subtle and do not allow a clear distinction between these two. Therefore, it is not currently possible to establish definitive differential diagnostic criteria that would allow differentiation. Paraneoplastic SSc should be separated from, more frequently reported, scleroderma-like syndrome associated with neoplasm. The latter does not usually feature immunological phenomena typical for SSc, such as high titres of autoantibodies to topoisomerase I (formerly termed anti-Scl-70-antibodies) or significant systemic involvement. In addition, recently, new antibodies have been linked with cancer-associated SSc, namely anti-RNA polymerase III (anti-RNAP III, also known as anti-POL) autoantibodies and autoantibodies to RNPC-3, a member of the minor spliceosome complex [11, 12]. Unfortunately, we were unable to test the patient for the presence of these autoantibodies due to the lack of available tests. It is noteworthy that many antineoplastic agents, such as docetaxel, paclitaxel, bleomycin, or carboplatin, may also trigger severe Raynaud's phenomenon, digital ischaemia, or exaggerated skin fibrosis reminiscent of SSc. In addition, certain immunotherapies may provoke autoimmunity, radiation may stimulate development of skin fibrosis, and bone marrow transplantation may induce SSc [13].

The exact pathogenesis of paraneoplastic background of some SSc cases is unknown. The most compelling hypothesis attributes SSc development to cancer-induced autoimmunity. It is also postulated that certain cancer cells may produce biologically active substances such as transforming growth factor $\beta$ (TGF- $\beta$ ), which has long been implicated in fibrotic pathology [14].

The clinical characteristics and laboratory findings in patients with paraneoplastic SSc are generally similar to those seen in idiopathic cases of the disease. However, disease onset in patients older than 50 years, asymmetrical Raynaud's phenomenon, a poor response to conventional treatments, and profound constitutional symptoms or weight loss are among the most com- mon features observed in patients with paraneoplastic SSc $[7,15]$. Several atypical features of the clinical and laboratory presentation of SSc raising the suspicion of an underlying tumour are shown in Table I.

Due to a slight decrease in mortality rate in patients with SSc, mainly as a consequence of improvement in renal crisis prevention and treatment, the risk of cancer development in SSc has been extensively investigated. The majority of studies suggest that patients with SSc have an elevated risk of all carcinomas; the risk increase is higher in men $[4,16]$ and related to older age at SSC onset [17]. Recognition of the association between SSC and malignancies further supports autoimmunity as necessary for initiating and maintaining the disease. In addition, it may serve as an indirect support for the current therapeutic guidelines that include immunosuppressive drugs $[3,18]$.

Along with identification of common features of patients with coexisting malignancy and SSc, efforts are being made to identify the causes of increased carcinogenesis in these individuals. Autoimmunity, persistent B-cell activation, chronic inflammatory process, and fibrosis may all result in malignant transformation in certain organ systems. Immunosuppressive therapies used to treat systemic sclerosis might also contribute to elevated risk of cancer. For example, it is well documented that an increased cumulative dose exposure to cyclophosphamide raises the risk of bladder cancer and haematological malignancies [19].

In the described patient the possibility of inducing SSc by chemotherapy is unlikely due to the presence of advanced skin and lung fibrosis, as well as vascular abnormalities at the moment of SSc diagnosis suggesting that the disease was more long-standing and most probably initiated before antineoplastic treatment.

Systemic sclerosis is a highly heterogeneous disease in its systemic manifestations; nearly $90 \%$ of SSc patients have some degree of gastrointestinal (GI) involvement. Although the oesophagus is the most frequently affected part of the GI tract, any part of the alimentary tract may be involved. The variety of signs and symptoms, including pain, dysphagia, vomiting, diarrhoea,

Table I. Clinical and laboratory features of systemic sclerosis suggestive of paraneoplastic aetiology (modified from [7])

\begin{tabular}{|ll|}
\hline 1. & Asymmetrical Raynaud's phenomenon unresponsive or poorly responsive to vasodilator drugs \\
\hline 2. & Severe or atypical systemic symptoms, e.g. malaise, fever, weight loss \\
\hline 3. & Sudden onset and rapid progression of clinical symptoms \\
\hline 4. & Late disease onset (after 50 years of age) \\
\hline 5. & Personal and/or family history of cancer \\
\hline 6. & Abnormal laboratory test results suggestive of cancer, e.g. anaemia, hypercalcaemia, hypergammaglobulinaemia \\
\hline 7. & Presence of anti-RNA polymerase III antibodies (anti-RNAP) \\
\hline
\end{tabular}


constipation, malabsorption, and weight loss, are often reminiscent of those seen in patients with GI malignancies. This similarity may result in delayed diagnosis of occult neoplasm in some cases, while in others it may prompt clinicians to carry out more timely GI tract diagnostics and ensure earlier detection of malignancy. Colorectal carcinoma has been rarely reported in association with SSc, and only exceptionally in the paraneoplastic context. Descriptions of paraneoplastic SSc secondary to other types of cancer are also uncommon. The current classification criteria do not allow distinction between paraneoplastic SSc from its idiopathic counterpart. The diagnosis is obtained from the aforementioned features, including the age of onset, the timely association of symptom onset with cancer, and response to antineoplastic/immunosuppressive treatment. SSc cases, in which marked regression of symptoms is not observed after surgical removal of the tumour or use of other efficacious antineoplastic modalities, should not be regarded as paraneoplastic.

\section{Conclusions}

There is a close temporal relationship between cancer diagnosis and SSc onset in a subset of patients, suggesting that there is a possible mechanistic link between these two diseases. Diagnostic workup plan in patients with suspected/diagnosed SSc should consider the possibility of cancer coexistence, or even a paraneoplastic background of the disease. Thorough malignancy screening in these patients may aid in early cancer detection, enabling therapeutic approach adjustment, and improving SSc outcome and patient survival. Raised oncological awareness is particularly recommended in patients with positive history of cancer, atypical course of SSc, and the presence of marked systemic symptoms or serological tumour markers.

All patients with paraneoplastic SSc should undergo thorough clinical and immunological follow-up because the increase in disease activity is likely to reflect the cancer recurrence. In all cases of SSc with atypical clinical presentation without underlying malignancy detected, continued vigilance for development of cancer in follow-up is advised.

The authors declare no conflict of interest.

\section{References}

1. Salazar G, Mayes MD. Genetics, epigenetics, and genomics of systemic sclerosis. Rheum Dis Clin North Am 2015; 41: 345366.

2. Abu-Shakra M, Guillemin F, Lee P. Cancer in systemic sclerosis. Arthritis Rheum 1993; 36: 46-54.
3. Airo' P, Ceribelli A, Cavazzana I, et al. Malignancies in Italian patients with systemic sclerosis positive for anti-RNA polymerase III antibodies. J Rheumatol 2011; 38: 1329-1334.

4. Onishi A, Sugiyama D, Kumagai $S$, et al. Cancer incidence in systemic sclerosis: meta-analysis of population-based cohort studies. Arthritis Rheum 2013; 65: 1913-1921.

5. Baijens LW, Manni J. Paraneoplastic syndromes in patients with primary malignancies of the head and neck. Four cases and a review of the literature. J Eur Arch Otorhinolaryngol 2006; 263: 32-36.

6. van den Hoogen F, Khanna D, Fransen J, et al. 2013 classification criteria for systemic sclerosis: an American College of Rheumatology/European League against Rheumatism collaborative initiative. Arthritis Rheum 2013; 65: 2737-2747.

7. Fam AG. Paraneoplastic rheumatic syndrome. Best Pract Res Clin Rheumatol 2000; 14: 515-533.

8. Świerkot J, Lewandowicz-Uszyńska A, Bogunia-Kubik K. Zespoły paranowotworowe a choroby reumatyczne. Postepy Hig Med Dosw 2014; 68: 944-954.

9. Nathanson L, Hall TC. Introduction: paraneoplastic syndromes. Semin Oncol 1997; 24: 265-268.

10. Barnes BE, Mawr B. Dermatomyositis and malignancy. A review of the literature. Ann Intern Med 1976; 84: 68-76.

11. Shah AA, Hummers LK, Casciola-Rosen L, et al. Examination of autoantibody status and clinical features associated with cancer risk and cancer-associated scleroderma. Arthritis Rheumatol 2015; 67: 1053-1061.

12. Shah AA, Xu G, Rosen A, et al. Anti-RNPC-3 antibodies as a marker of cancer-associated scleroderma. Arthritis Rheumatol 2017; 69: 1306-1312.

13. Kupfer I, Balguerie X, Courville P, et al. Scleroderma-like cutaneous lesions induced by paclitaxel: a case study. J Am Acad Dermatol 2003; 48: 279-281.

14. Fujii T, Mimori T, Kimura N, et al. Pseudoscleroderma associated with transforming growth factor beta 1-producing advanced gastric carcinoma: comment on the article by Varga. Arthritis Rheum 2003; 48: 1766-1767.

15. Leandro MJ, Isenberg DA. Rheumatic diseases and malignancy - is there an association? Scand J Rheumatol 2001; 30: 185188.

16. Olesen AB, Svaerke C, Farkas DK, et al. Systemic sclerosis and the risk of cancer: a nationwide population-based cohort study. Br J Dermatol 2010; 163: 800-806.

17. Shah AA, Casciola-Rosen L. Cancer and scleroderma: a paraneoplastic disease with implications for malignancy screening. Curr Opin Rheumatol 2015; 27: 563-570.

18. Shah AA, Rosen A, Hummers L, et al. Close temporal relationship between onset of cancer and scleroderma in patients with RNA polymerase I/III antibodies. Arthritis Rheum 2010; 62: 2787-2795.

19. Zeineddine N, Khoury LE, Mosak J. Systemic Sclerosis and Malignancy: A Review of Current Data. J Clin Med Res 2016; 8: 625-632. 